\title{
The design of conservative finite element discretisations for the vectorial modified $K d V$ equation
}

Article

Accepted Version

Creative Commons: Attribution-Noncommercial-No Derivative Works 4.0

Jackaman, J., Papamikos, G. and Pryer, T. (2019) The design of conservative finite element discretisations for the vectorial modified KdV equation. Applied Numerical Mathematics, 137. pp. 230-251. ISSN 0168-9274 doi:

https://doi.org/10.1016/j.apnum.2018.10.006 Available at https://centaur.reading.ac.uk/81475/

It is advisable to refer to the publisher's version if you intend to cite from the work. See Guidance on citing.

To link to this article DOI: http://dx.doi.org/10.1016/j.apnum.2018.10.006

Publisher: Elsevier

All outputs in CentAUR are protected by Intellectual Property Rights law, including copyright law. Copyright and IPR is retained by the creators or other copyright holders. Terms and conditions for use of this material are defined in the End User Agreement.

www.reading.ac.uk/centaur 
Central Archive at the University of Reading

Reading's research outputs online 


\title{
An exponential model of urban geometry for use in radiative transfer applications
}

\author{
3 Robin J. Hogan
}

5 the date of receipt and acceptance should be inserted later

Abstract In radiative transfer schemes for urban areas it is common to approximate urban geometry by infinitely long streets of constant width, or other very idealized forms. For solar and thermal-infrared radiative transfer applications, we argue that horizontal urban geometry is described uniquely by the probability distribution of wall-to-wall separation distances. The analysis of building layout from contrasting neighbourhoods in London and Los Angeles reveals this function to be well fitted by an exponential distribution. Compared to the infinite-street model, this exponential model of urban geometry is found to lead to a significantly more accurate description of the rates of exchange of radiation between the sky, the walls and the streets of an urban canopy.

Keywords Radiative transfer - Street canyon - Urban Meteorology

\section{Introduction}

With the increasing urbanization of the world's population (United Nations, 2015) and the ever higher resolution of weather and climate models, there is a need to improve the fidelity with which urban areas are represented in such models. This is a pre-requisite for better prediction of the urban-heat-island effect and its impact on both city inhabitants at street level and the atmosphere downstream (e.g. Grimmond et al., 2010). The complexity and variety of urban structure, with streets of different widths, intersections, parking areas and parks, presents a challenge for modelling both the exchange of solar and thermal-infrared radiation, and the turbulent transport of heat, momentum and pollutants. Inevitably the geometry must be simplified in order that processes can be represented efficiently, and the complexity needs to be

R. J. Hogan

European Centre for Medium Range Weather Forecasts, Shinfield Park, Reading, RG2 9AX, United Kingdom. E-mail: r.j.hogan@ecmwf.int.

Additional affiliation: Department of Meteorology, University of Reading, United Kingdom. 
commensurate with the small number of parameters that are typically available to describe variations in urban geometry within regional and global models.

In the case of urban radiation schemes, a common simplification is to consider an infinitely long street of fixed width with random azimuthal orientation relative to the sun (e.g. Masson, 2000; Harman et al., 2004; Li et al., 2016). In the horizontal plane, the geometry of this 'infinite-street model' can be described by just two parameters: the fraction of built-up area occupied by buildings, $\lambda_{P}$, and the street width, $W$. These are accompanied by the building height, $H$, which is typically assumed constant. From these parameters, several radiative exchange factors (called shape factors by Harman et al., 2004) can be computed such as the fraction of direct (i.e. unscattered) solar radiation that penetrates down to street level, and the fraction of diffuse radiation emitted or scattered by the walls that then intercepts another wall. Somewhat more sophisticated descriptions of horizontal urban geometry have been proposed, such as a regular array of square-based blocks with intersections at regular intervals (Kondo et al., 2005), but in the intercomparison of urban models by Grimmond et al. (2010), only six of the 33 models described horizontal urban geometry by anything more sophisticated than an infinite-street canyon. A number of models now incorporate radiative interaction with buildings of different height (e.g. Martilli et al., 2002; Schubert et al., 2012) and street trees (Krayenhoff et al., 2014; Redon et al., 2017), but they are still typically underpinned by the infinite-street assumption. Clearly there is a need to test and if necessary improve this assumption.

In this paper an alternative 'exponential model' for characterizing horizontal urban geometry is proposed and evaluated. It uses the same number of parameters as the infinite-street model, yet has the potential to describe the much more complex geometry of real cities. Section 2 demonstrates that for the purposes of radiation, horizontal building layout may be described uniquely by the probability distribution of wall-to-wall separation distances, and it is shown how the radiative exchange factors may be derived from this function. Section 3 describes how the infinite-street model may be posed in terms of this probability distribution, and confirms that the resulting formulas for the radiative exchange factors match those in the literature. Section 4 introduces the exponential model, and derives alternative formulas for these factors. Then in Sect. 5, probability distributions are derived from real building distributions in residential and commercial parts of London and Los Angeles, and used to evaluate the accuracy of the infinite-street and exponential models in terms of how well they predict the 'true' radiative exchange factors. It is important to stress that radiative exchange factors provide a convenient way of evaluating the validity of the two assumptions for radiative transfer, but do not themselves represent the important effects of street trees, buildings of different heights, or absorption by air in the urban canopy. In Sect. 6 we discuss how the exponential model could be incorporated into more sophisticated schemes that do capture these effects.

\section{Urban geometry in terms of probability distributions}

We here consider how best to describe the horizontal distribution of buildings, so for simplicity we assume that all buildings are the same height $(H)$ with flat roofs 
(a) Wall-to-wall distances

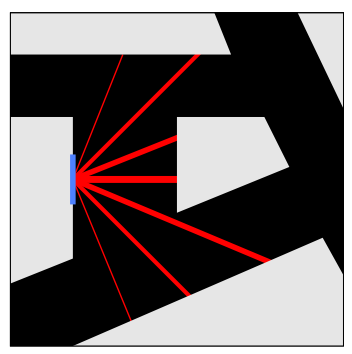

(b) Ground-to-wall distances

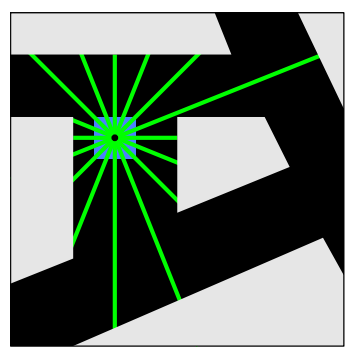

(c) Relationship

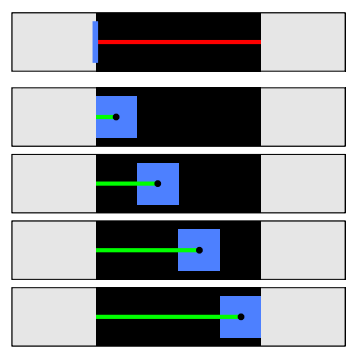

Fig. 1 Plan view of a small section of an urban canopy illustrating the definitions of the probability distributions $p_{w w}$ and $p_{g_{w}}$. (a) The red lines depict wall-to-wall distances $x$ originating from a small vertical strip of wall (in blue); the probability distribution of $x$ from all such strips is denoted $p_{w w}(x)$. The thickness of each line is proportional to the angle subtended by the strip in that particular direction. (b) The green lines depict the ground-to-wall distances $x$ from a small facet of the ground (depicted by the blue square); the probability distribution of $x$ from all such facets is denoted $p_{g w}(x)$. (c) Illustration of the property that a single wall-to-wall distance $x^{\prime}$ (the red line) is associated with ground-to-wall distances $x$ in the range $0<x<x^{\prime}$ (shown by the four green lines), leading to the relationship between the two probability distributions given by (1). In each panel the buildings are shown in light grey and the ground in black.

and vertical walls. Consider diffuse radiation emitted or scattered from a thin vertical strip of wall in a particular azimuthal direction. Since radiation travels in straight lines, the probability of it being intercepted by another wall, rather than escaping to the atmosphere above or striking the ground, is a function of the distance between the two walls and their height. To determine the fraction of diffuse radiation emitted isotropically from all the walls in the neighbourhood that intercept another wall, we need to consider $p_{w w}(x)$, the probability distribution of wall-to-wall horizontal separation distances, $x$, considering all possible azimuth angles. Thus, a pedestrian walking away from a randomly selected point on a wall in a random direction has a probability $p_{w w}(x) \mathrm{d} x$ of encountering another wall after walking a distance between $x$ and $x+\mathrm{d} x$. This is illustrated in Fig. 1a, where the variable thickness of the red lines highlights that the probability of radiation being emitted or scattered from the strip in a particular azimuthal direction $\phi$ varies as the cosine of the angle between $\phi$ and the wall normal.

For computing radiative exchanges between the ground (or street) and the walls, we need instead $p_{g w}(x)$, the probability distribution of ground-to-wall horizontal distances within the urban canopy at all possible azimuth angles. In this case, a pedestrian walking in a random direction from a randomly selected point at ground level has a probability $p_{g w}(x) \mathrm{d} x$ of encountering a wall after walking a distance between $x$ and $x+\mathrm{d} x$, as illustrated in Fig. 1b.

There is a unique relationship between $p_{w w}$ and $p_{g w}$, since as shown in Fig. 1c, any single wall-to-wall distance $x^{\prime}$ can be split into many ground-to-wall distances $x$, where $x<x^{\prime}$. Therefore, the probability density $p_{g w}(x)$ of a particular ground-to-wall 
distance $x$ is proportional to the probability of $x^{\prime}>x$ :

$$
\begin{aligned}
& p_{g w}^{\prime}(x)=\int_{x}^{\infty} p_{w w}\left(x^{\prime}\right) \mathrm{d} x^{\prime}, \\
& p_{g w}(x)=p_{g w}^{\prime}(x) / \int_{0}^{\infty} p_{g w}^{\prime}(x) \mathrm{d} x,
\end{aligned}
$$

where (1b) normalizes the 'raw' distribution $p_{g w}^{\prime}$ such that the normalized distribution $p_{g w}$ integrates to unity.

From these two probability distributions, and assuming a vacuum, we may compute radiative exchange factors, $F_{i j}$, which denote the fraction of radiation originating from source $i$ that illuminates destination $j$, where we assign the ground, wall and 'sky' facets the subscripts $g, w$ and $s$, respectively. We add an additional possible source subscript ' 0 ' denoting direct solar radiation from the sky facet, whereas all other sources are diffuse. Some authors (e.g. Masson, 2000; Li et al., 2016) refer to $F_{i j}$ as 'sky view factors', but we avoid this term as it is more commonly used in the literature to refer to the sky fraction viewed by an observer at a specific point on a facet (e.g. Johnson and Watson, 1984), rather than integrated over all points on a facet as signified by $F_{i j}$. All the equations for the $F_{i j}$ exchange factors that follow involve integration over one of the two probability distributions above, and may be applied either analytically to parametric models for the probability distributions (as in Sects. 3 and 4), or numerically to probability distributions derived from real building layouts (as in Sect. 5).

Consider first direct solar radiation, which travels horizontally a distance $x_{0}$ between the top and bottom of the urban canopy given by

$$
x_{0}=H \tan \theta_{0},
$$

where $\theta_{0}$ is the solar zenith angle. This means that direct radiation entering the top of the canopy at a particular point only penetrates to ground level if the nearest wall in the azimuthal direction of the radiation is at least a distance $x_{0}$ away. Therefore, the fraction $F_{0 g}$ of direct radiation just below canopy top that penetrates down to ground level without being intercepted by a wall is

$$
F_{0 g}=\int_{x_{0}}^{\infty} p_{g w}(x) \mathrm{d} x .
$$

Any direct radiation just below canopy top that does not reach the ground must be intercepted by a wall, so $F_{0 w}=1-F_{0 g}$.

The fraction of diffuse radiation emitted or scattered from ground level that is intercepted by a wall is

$$
F_{g w}=\int_{0}^{\infty} p_{g w}(x) f_{g w}(H / x) \mathrm{d} x,
$$

where $f_{g w}(H / x)$ is the fraction of diffuse radiation emitted from a small horizontal area at ground level into the quadrant towards a wall of height $H$ a distance $x$ away, which is intercepted by the wall. To derive an expression for $f_{g w}$, consider the beam of radiation emitted from point $A$ in Fig. 2a that intercepts the wall at point 


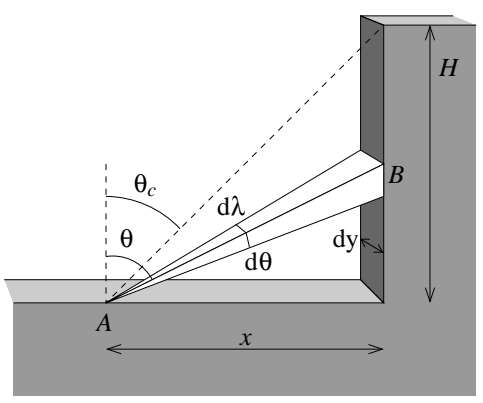

(a) Ground-to-wall geometry

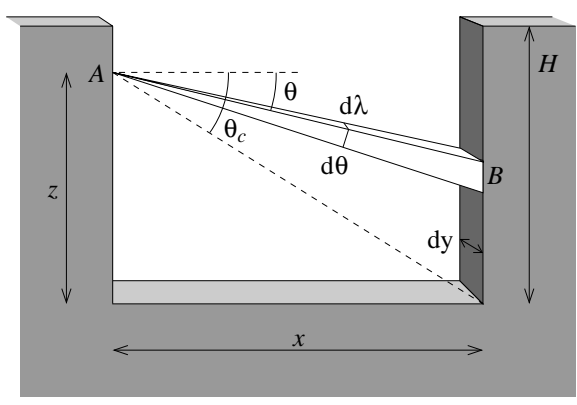

(b) Wall-to-wall geometry

Fig. 2 Schematic of thin slices through an urban area illustrating the geometry used in Sect. 2 to compute the fraction of diffuse radiation emitted or scattered from (a) the ground and (b) a wall, which subsequently intercepts a wall. If the wall at $B$ has an azimuthal orientation such that the light beam strikes it at an oblique azimuthal angle, then note that elemental length $\mathrm{d} y$ is the horizontal width of the beam, not the horizontal length of the wall at $B$ that is illuminated by the beam (which could be larger).

$B$. If the emission is isotropic then the radiative power in this infinitesimally narrow beam is proportional to the solid angle $\mathrm{d} \lambda \mathrm{d} \theta$, multiplied by $\cos \theta$ to account for the dependence on $\theta$ of the angle subtended by the small horizontal area at $A$ to an observer at $B$. From geometry we have $\mathrm{d} \lambda=\sin \theta \mathrm{d} y / x$, so the radiative power is proportional to $\sin \theta \cos \theta \mathrm{d} \theta \mathrm{d} y / x$. The fraction of radiative power emitted into the quadrant $0<\theta<\pi / 2$ that intercepts the wall is therefore given by

$$
f_{g w}(H / x)=\frac{\int_{\theta_{c}}^{\pi / 2} \sin \theta \cos \theta \mathrm{d} \theta}{\int_{0}^{\pi / 2} \sin \theta \cos \theta \mathrm{d} \theta},
$$

where the $\mathrm{d} y / x$ term is not a function of $\theta$ so cancels between numerator and denominator. The critical zenith angle beyond which the beam starts to intersect the building is $\theta_{c}=\tan ^{-1}(H / x)$, so (5) simplifies to

$$
f_{g w}(H / x)=\frac{1}{1+(x / H)^{2}} .
$$

The fraction of diffuse radiation emanating from the ground that escapes to the sky is simply the fraction not intercepted by the walls, so we can write $F_{g s}=1-F_{g w}$, or equivalently

$$
F_{g s}=\int_{0}^{\infty} p_{g w}(x) f_{g s}(H / x) \mathrm{d} x,
$$

where

$$
f_{g s}(H / x)=1-f_{g w}=\frac{1}{1+(H / x)^{2}} .
$$

Moreover, the symmetry of the problem with respect to the sky and the ground implies that for diffuse radiation emanating from the sky we can write $F_{s g}=F_{g s}$ and $F_{s w}=F_{g w}$. 
The fraction of diffuse radiation emitted or scattered from a wall that then encounters another wall is a function of the wall-to-wall probability distribution,

$$
F_{w w}=\int_{0}^{\infty} p_{w w}(x) f_{w w}(H / x) \mathrm{d} x,
$$

where $f_{w w}(H / x)$ is the fraction of diffuse radiation emitted from a small width of wall (but all heights up the wall) that intercepts another wall at distance $x$ given that the buildings are of height $H$. This calculation is more involved as we need to integrate over all emission heights. We define $g_{w w}(z / x)$ as the fraction of diffuse radiation emitted into the downward quadrant from a small area of wall at height $z$ that intercepts the other wall at distance $x$, rather than the ground. Consider the infinitesimally narrow beam of radiation emitted from point $A$ in Fig. $2 \mathrm{~b}$ that arrives at point $B$. The radiative power in the beam is again proportional to $\cos \theta \mathrm{d} \lambda \mathrm{d} \theta$, where $\theta$ is now the angle relative a horizontal line emanating from the wall in the direction of $B$ (not necessarily the normal to the wall since the wall elements at $A$ and $B$ need not be azimuthally parallel to each other). This time $\mathrm{d} \lambda=\cos \theta \mathrm{d} y / x$, so the radiative power is proportional to $\cos ^{2} \theta \mathrm{d} \theta \mathrm{d} y / x$, leading to

$$
g_{w w}(z / x)=\frac{\int_{0}^{\theta_{c}} \cos ^{2} \theta \mathrm{d} \theta}{\int_{0}^{\pi / 2} \cos ^{2} \theta \mathrm{d} \theta}=\frac{2}{\pi}\left[\tan ^{-1} \frac{z}{x}+\left(2+\frac{z^{2}}{x^{2}}+\frac{x^{2}}{z^{2}}\right)^{-1 / 2}\right],
$$

where the critical angle is $\theta_{c}=\tan ^{-1}(z / x)$. Integrating $g_{w w}$ over all heights up the wall yields

$$
f_{w w}=\frac{1}{H} \int_{0}^{H} g_{w w} \mathrm{~d} z=\frac{2}{\pi} \tan ^{-1} \frac{H}{x} .
$$

Note that here we have considered only radiation emitted into the downward quadrant $(0<\theta<\pi / 2$ in Fig. $2 b)$, but the symmetry of the problem means that the fraction of diffuse radiation emitted from a wall into the equivalent upward quadrant that intercepts another wall is the same, so (11) is valid for radiation emitted into either quadrant.

In assessing different models for urban geometry, we shall use the equations in this section to evaluate how well the models predict the exchange factors $F_{0 g}, F_{g s}$ and $F_{w w}$. The other exchange factors are unique functions of these three; we have already seen that $F_{0 w}=1-F_{0 g}, F_{g w}=1-F_{g s}, F_{s g}=F_{g s}$ and $F_{s w}=F_{g w}$. Furthermore, the diffuse radiation emanating from a wall that does not hit another wall must be evenly divided between the sky and the ground, so $F_{w g}=F_{w s}=\left(1-F_{w w}\right) / 2$.

\section{The infinite street canyon model}

To demonstrate how the general approach in terms of probability distributions may be applied to a specific geometry, we consider the case of infinitely long street canyons of width $W$, a common assumption as discussed in Sect. 1. The wall-to-wall distance in the horizontal plane is then given by

$$
x=W / \cos \phi,
$$


where $\phi$ is the azimuthal direction from the wall normal such that $\phi=0$ is the direction of shortest distance across the street, and $\phi=\pi / 2$ is directed along the street. If the fraction of the urban area occupied by buildings is $\lambda_{p}$ then the distance between adjacent streets in direction $\phi$ is $S=W /\left[\left(1-\lambda_{p}\right) \cos \phi\right]$. The probability of wall-towall separation distances lying in the range $x$ to $x+\mathrm{d} x$ is then equal to the probability of azimuthal angles lying in the range $\phi$ to $\phi+\mathrm{d} \phi$, i.e.

$$
p_{w w}(x) \mathrm{d} x=p(\phi) \mathrm{d} \phi .
$$

Each azimuthal street orientation is equally likely, implying that $p(\phi)$ should be constant, but from the definition of $S$ we see that the distance between streets in direction $\phi$ is proportional to $1 / \cos \phi$, implying that the probability density of streets in direction $\phi$ is actually $p(\phi)=\cos \phi$. Differentiating (12) and substituting into (13) yields $p_{w w}=\cos ^{3} \phi /(W \sin \phi)$. Using (12) to express this in terms of $W$ and $x$, and recognizing that this expression is only valid for distances larger than the street width, yields

$$
p_{w w}(x, W)=\left\{\begin{array}{lr}
0: & x \leq W \\
\frac{W^{2}}{x^{2}}\left(x^{2}-W^{2}\right)^{-1 / 2}: & x>W .
\end{array}\right.
$$

The probability distribution of ground-to-wall distances is found by applying (1) to (14), to obtain

$$
p_{g w}(x, W)=\frac{2}{\pi W}\left(1-\sqrt{1-\frac{\min (W, x)^{2}}{x^{2}}}\right) .
$$

The radiative exchange factors may now be derived. Applying (3) to (15) we obtain

$$
F_{0 g}=\frac{2}{\pi}\left[\frac{Y-x_{0}}{W}+\tan ^{-1} \frac{W}{Y}\right],
$$

where $Y=\max \left(x_{0}^{2}-W^{2}, 0\right)^{1 / 2}$. This is mathematically equivalent to Eq. 13 of Masson (2000). Similarly we apply (7) and (8) to (15), and (9) and (11) to (14), to obtain (after considerable manipulation)

$$
\begin{gathered}
F_{g s}=\sqrt{\frac{H^{2}}{W^{2}}+1}-\frac{H}{W} \\
F_{w w}=\sqrt{\frac{W^{2}}{H^{2}}+1}-\frac{W}{H},
\end{gathered}
$$

which match the relations found previously (e.g. Sparrow and Cess, 1970; Noilhan, 1981; Masson, 2000; Harman et al., 2004). 


\section{The exponential model}

In this section an alternative model for horizontal urban geometry is proposed in which the two probability distributions are assumed to follow an exponential distribution,

$$
p_{w w}(x)=p_{g w}(x)=\exp (-x / X) / X,
$$

which satisfies the relationship between the two distributions given by (1). This distribution was assumed for the separation of trees in the forest radiative-transfer scheme of Hogan et al. (2018). The validity of the exponential model for urban areas is evaluated using real building layouts in the next section. As with the infinite-street model, only one parameter is used to characterize the distribution, in this case the 'e-folding' building separation $X$. Since $X$ is also the mean value of the exponential distribution, it can be interpreted physically as the mean wall-to-wall distance considering all directions (i.e. the mean length of the red lines in Fig. 1a) or the mean ground-to-wall distance (i.e. the mean length of the green lines in Fig. 1b). However, when fitting an exponential distribution to the geometry of real cities, the method described in Sect. 5 should be used rather than simply setting $X$ to the observed mean wall-to-wall separation distance.

The radiative exchange factors may again be derived by applying the integrals in Sect. 2. The penetration of direct radiation to ground level also has an exponential form,

$$
F_{0 g}=\exp \left(-x_{0} / X\right)
$$

where $x_{0}$ is given by (2). This is essentially the Beer-Lambert law, and indicates that the penetration of direct radiation through an urban scene obeying the exponential model is the same as the penetration of direct radiation through a turbid medium with an extinction coefficient that does not vary with height.

The radiative exchange factors for diffuse radiation have a more complex form,

$$
\begin{aligned}
F_{g s} & =1+\zeta\left[\cos \zeta\left(\mathrm{Si} \zeta-\frac{\pi}{2}\right)-\sin \zeta \mathrm{Ci} \zeta\right] \\
F_{w w} & =1+\frac{2}{\pi}\left[\cos \zeta\left(\mathrm{Si} \zeta-\frac{\pi}{2}\right)-\sin \zeta \mathrm{Ci} \zeta\right]=1+\frac{2}{\pi \zeta}\left(F_{g s}-1\right),
\end{aligned}
$$

where $\zeta=H / X, \operatorname{Si}(\cdot)$ is the sine integral and $\mathrm{Ci}(\cdot)$ is the cosine integral. In an operational model, these exchange factors could be implemented efficiently as onedimensional look-up tables or Padé approximants.

Figure 3 compares the radiative exchange factors between the infinite-street model and the exponential model, as a function of the ratio of total wall area $A_{w}$ to total ground area $A_{g}$. In the case of the infinite street, the ratio is

$$
A_{w} / A_{g}=2 H / W,
$$

since there are two walls for every street. For the exponential model, we apply energy conservation principles: if each surface of the urban area is at the same temperature (including the sky) and has an emissivity of unity then the energy emitted from a surface equals the energy received. For the walls this leads to

$$
A_{w} B=2 A_{g} F_{g w} B+A_{w} F_{w w} B,
$$



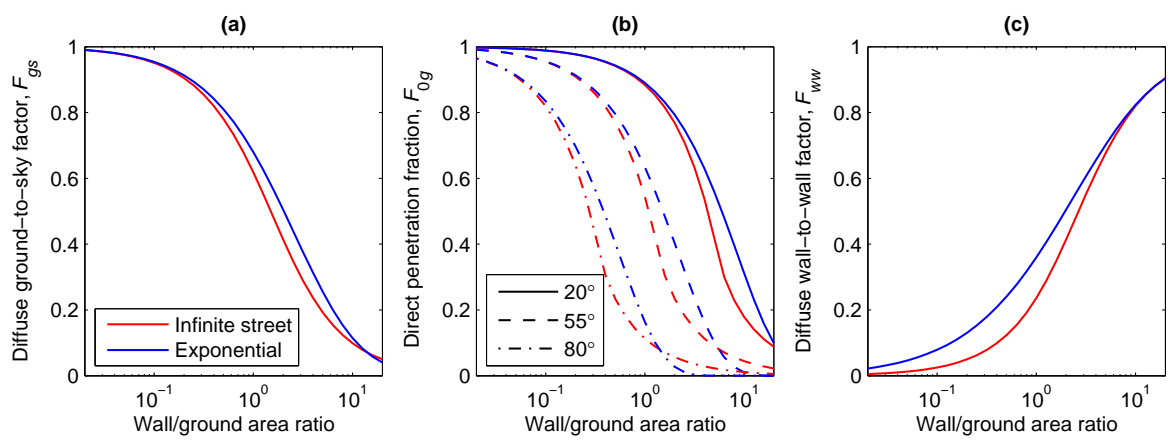

Fig. 3 Comparison of radiative exchange factors between the infinite-street model and the exponential model. The wall/ground area ratio, $A_{w} / A_{g}$, is defined in terms of the parameters of the two models by (23) and (25), and varies in the range $0.26-1.4$ for the scenes analyzed in Sect. 5. Panel b shows $F_{0 g}$ for the three different solar zenith angles indicated in the legend.

where $B$ is the power emitted per unit area (in $\mathrm{W} \mathrm{m}^{-2}$ ), the term on the left-hand side is the total power emitted from the walls, the first term on the right is the power received at the walls from the ground and sky (which is the same) and the second term on the right is the power received from other walls. Combining with (22), and noting again that $F_{g w}=1-F_{g s}$, we obtain

$$
A_{w} / A_{g}=\pi H / X
$$

Equations 23 and 25 enable the two models to be plotted on the same axes in Fig. 3. These equations imply that the parameters $W$ and $X$ could be fitted to real cities from measurements of $A_{w} / A_{g}$, but in practice the wall area $A_{w}$ is a somewhat ill-defined quantity in that it depends on the resolution of the measurements, and some buildings have fine-scale details that are not important for radiative exchange. Therefore we prefer the approach taken below, where $W$ and $X$ are fitted such that one of the radiative exchange factors is predicted exactly, and the validity of the model is assessed by how well the other factors are predicted.

\section{Analysis of real cities}

Here, the wall-to-wall and ground-to-wall probability distribution functions are computed for real cities, from which the radiative exchange factors are calculated numerically. This enables us to evaluate the different approximations to urban geometry described in Sects. 3 and 4. Building outlines and heights have been obtained for two cities, London and Los Angeles, and Fig. 4 depicts four $3 \mathrm{~km} \times 3 \mathrm{~km}$ scenes in which the buildings have been rendered on grid with a horizontal resolution of $\Delta x=2 \mathrm{~m}$. The scenes have been chosen to be very contrasting: the streets in Central London have an irregular layout and a range of different widths, the Residential London scene consists of a patchwork of rows of terraced housing, Downtown Los 
Angeles consists of a grid layout with large buildings in each block, and the Residential Los Angeles scene consists of a grid layout but with many small detached houses in each block. In the case of Central London, the location of the River Thames has been added manually using Google Maps imagery. The choice of $3 \times 3 \mathrm{~km}$ domains is a compromise between the need for a scene to be large enough to sample streets of different orientation and to minimize sampling noise in the probability distributions, but small enough that the 'character' of the building layout is similar everywhere in a scene. The datasets do not contain information about the location of trees, which are known to be important for urban radiative transfer (Grimmond et al., 2010), but in Sect. 6 we discuss how our results could be incorporated into a more sophisticated urban radiation scheme that includes urban vegetation.

Before analyzing the building spacings, a question arises as to how to treat large open areas such as rivers and parks. Most global weather and climate models treat each gridbox of the surface by a number of tiles of different types, including open water, grassland and forest, in addition to urban. When green areas are small, such as gardens and small parks, their associated radiative and turbulent fluxes are significantly affected by nearby buildings and they are best treated as part of the urban tile. When they are large and most of their area is a long distance from the nearest building, it is more appropriate to treat them as a separate tile. However, there is no consensus on the size of the green space at which the transition should take place. We do not attempt to answer this question in this paper, but rather examine its effect on the probability distributions.

Contiguous regions of the domain that are at least 0.5 hectares in area and at least $20 \mathrm{~m}$ from the nearest building or river pixel have been identified automatically. Google Maps was then used to manually determine whether each such region is a parking area or plaza, a park, or a built-up surface not frequented by pedestrians (such as a railway or major highway). Parking areas and plazas are assigned to the same category as streets, while the other two are treated separately as shown in Fig. 4. The rationale of keeping major highways separate is that one of the main purposes of an urban model is to predict the conditions experienced by pedestrians at street level, but the impact of this decision is investigated below. The first three rows of Table 1 list some basic properties of the four scenes.

Each gridded scene has been analyzed in four azimuthal directions, as illustrated in Fig. 5. Considering first the north-south and east-west directions in Figs. 5a and $5 b$, the scene is analyzed in one-dimensional strips of width $\Delta x$, and in each strip the transitions from building-to-street and street-to-building are identified. From these the contiguous spans of the street category are identified, shown by the red lines. Note that in the first analysis any spans that include rivers, parks, railways or major highways are excluded, but in the second analysis towards the end of this section only those including rivers are excluded. Thus we may build up the probability distribution of wall-to-wall separation distances, $p_{w w}$, at the resolution of the grid (in this case $2 \mathrm{~m}$ ). A similar analysis of the diagonal strips (Figs. $5 \mathrm{c}$ and $5 \mathrm{~d}$ ) produces a probability distribution with a grid spacing $\sqrt{2}$ times larger. This is interpolated back on to the 2-m grid and averaged with the first $p_{w w}$ estimate, using a weighting that accounts for the fact that each diagonal strip is a factor of $\sqrt{2}$ times narrower. The probability distribution of ground-to-wall separation distances, $p_{g w}$, is computed by applying (1) 

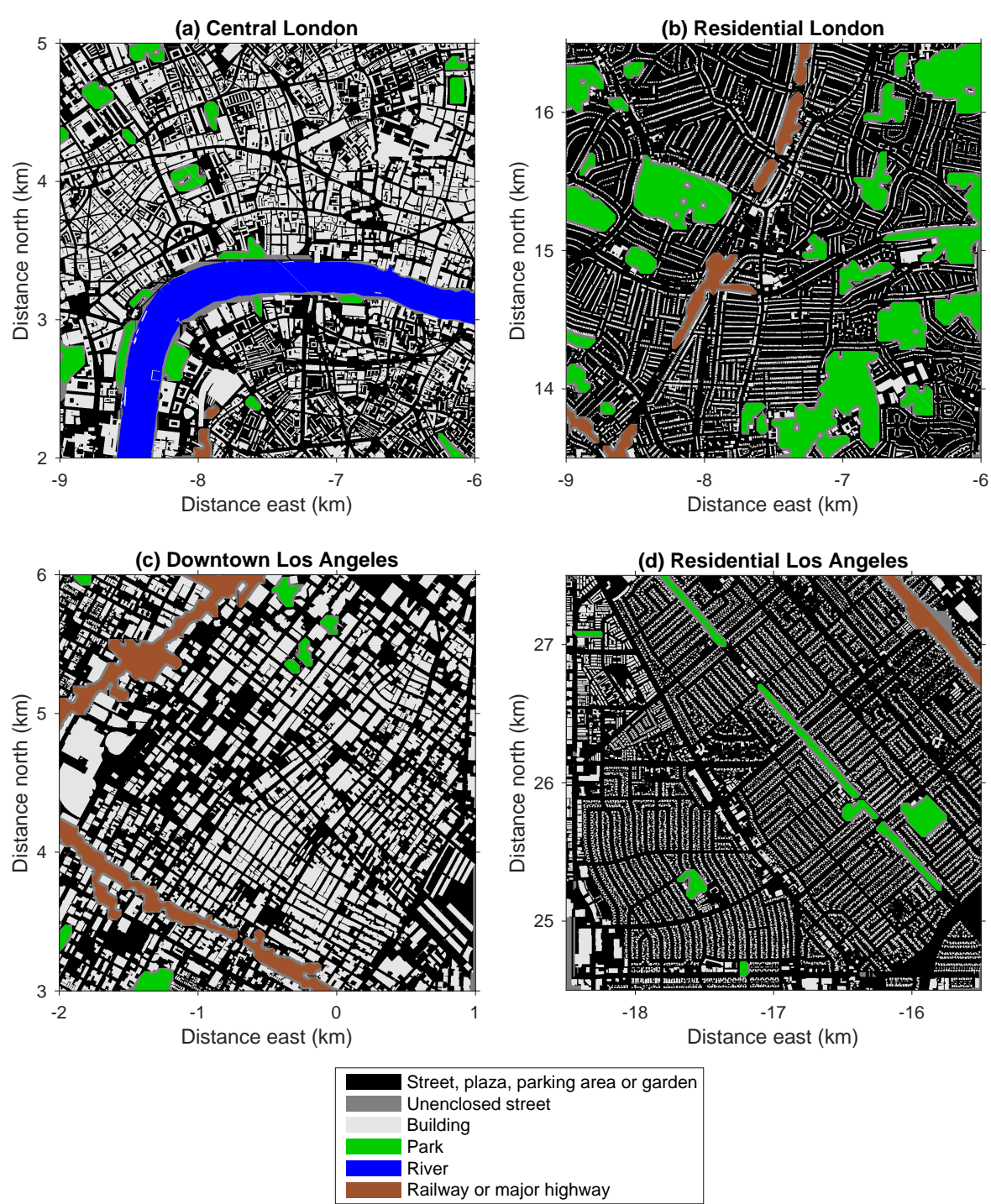

Fig. 4 Building layouts for four contrasting neighbourhoods of London and Los Angeles. The axes in the top two panels are indicated relative to a point $51.45^{\circ} \mathrm{N}, 0^{\circ} \mathrm{E}$. The axes in the bottom two panels are indicated relative to a point $34^{\circ} \mathrm{N}, 118.25^{\circ} \mathrm{W}$. Panel b shows the Palmers Green area of north London, while Panel d shows the Panorama City area of Los Angeles.

numerically to $p_{w w}$. A small fraction of the street pixels in the scene, particularly in the corners and at the borders of parks, are not sampled by this analysis in any of the four directions due to them not lying between two buildings in the directions considered; these are shown in dark grey in Fig. 4.

Care should be taken in applying the strip method of Fig. 5 to parts of several North American cities if all the streets are preferentially aligned along two of the strip 
Table 1 Numerical properties of the four scenes depicted in Fig. 4. 'Urban fraction' is the fraction of the domain occupied by streets, plazas, parking areas, gardens or buildings, and 'building fraction' is the fraction of this urban area that is occupied by buildings. The street width $(W)$ of the infinite-street model and the e-folding separation $(X)$ of the exponential model have each been fitted to ensure that these models predict the ground-to-sky factor $\left(F_{g s}\right)$ exactly. Therefore, the errors presented in the table are only for the predicted wall-to-wall factor $\left(F_{w w}\right)$.

\begin{tabular}{lcccc}
\hline Property & $\begin{array}{c}\text { Central } \\
\text { London }\end{array}$ & $\begin{array}{c}\text { Residential } \\
\text { London }\end{array}$ & $\begin{array}{c}\text { Downtown } \\
\text { Los Angeles }\end{array}$ & $\begin{array}{c}\text { Residential } \\
\text { Los Angeles }\end{array}$ \\
\hline Mean building height $H(\mathrm{~m})$ & 17.0 & 6.6 & 19.7 & 4.8 \\
Urban fraction & 0.88 & 0.83 & 0.94 & 0.97 \\
Building fraction $\lambda_{p}$ & 0.47 & 0.20 & 0.43 & 0.25 \\
Diffuse ground-to-sky factor $F_{g s}$ & 0.60 & 0.84 & 0.66 & 0.88 \\
Diffuse wall-to-wall factor $F_{w w}$ & 0.39 & 0.16 & 0.37 & 0.15 \\
Fitted street width $W(\mathrm{~m})$ & 32.0 & 38.8 & 46.4 & 36.0 \\
Fitted e-folding separation $X(\mathrm{~m})$ & 38.2 & 52.8 & 56.9 & 50.1 \\
Error in $F_{w w}$ from infinite-street model & $-36 \%$ & $-48 \%$ & $-45 \%$ & $-55 \%$ \\
Error in $F_{w w}$ from exponential model & $+10 \%$ & $+27 \%$ & $+3 \%$ & $+18 \%$ \\
\hline
\end{tabular}

a

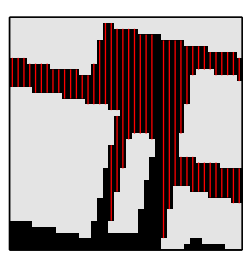

b

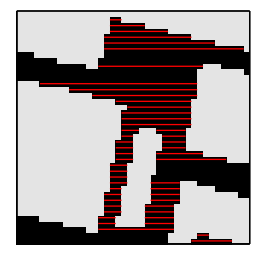

C

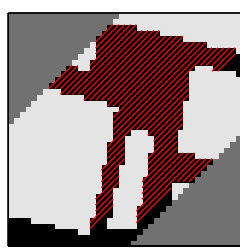

d

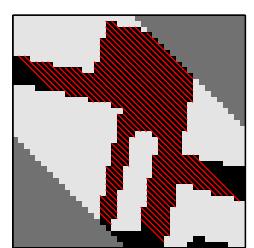

Fig. 5 Illustration of how the wall-to-wall probability distribution, $p_{w w}(x)$, is computed numerically from a digitized building layout, in this case considering an $80 \times 80-\mathrm{m}$ subset of Fig. 4 a at a resolution of $2 \mathrm{~m}$. The scene is analyzed in four directions: (a) north-south, (b) east-west, (c) northeast-southwest and (d) northwest-southeast, and $p_{w w}(x)$ is constructed from the valid wall-to-wall distances $x$ depicted by the red lines in each panel. The dark grey triangles in panels $\mathrm{c}$ and $\mathrm{d}$ are excluded from consideration since they are too small to contain the larger $x$ values so could skew the distribution towards small $x$.

directions. One approach to mitigate potential biases would be to rotate the building polygon data by several different angles before discretizing to a grid and performing the strip analysis. There is some preference for northwest-southeast and northeastsouthwest street orientation in the Residential Los Angeles scene (Fig. 4d), but we find below that the results for this scene are very similar to those from the Residential London scene (Fig. 4b), which has a much more random street orientation.

The black lines in Figs. 6a-6h depict the probability distributions derived from the four scenes. From these the various radiative exchange factors have been calculated numerically. The black lines in Fig. 6i-6k depict $F_{0 g}$ as a function of $\cos \theta_{0}$, while the diffuse factors $F_{g s}$ and $F_{w w}$ are shown in Table 1. Building height appears to be the dominant factor controlling radiative exchange, with the two downtown scenes having much lower penetrations of direct and diffuse radiation between sky and ground than the two residential areas.

We next investigate how well these distributions are fitted by the infinite-street and exponential models. The question arises of how best to fit the characteristic lengths 

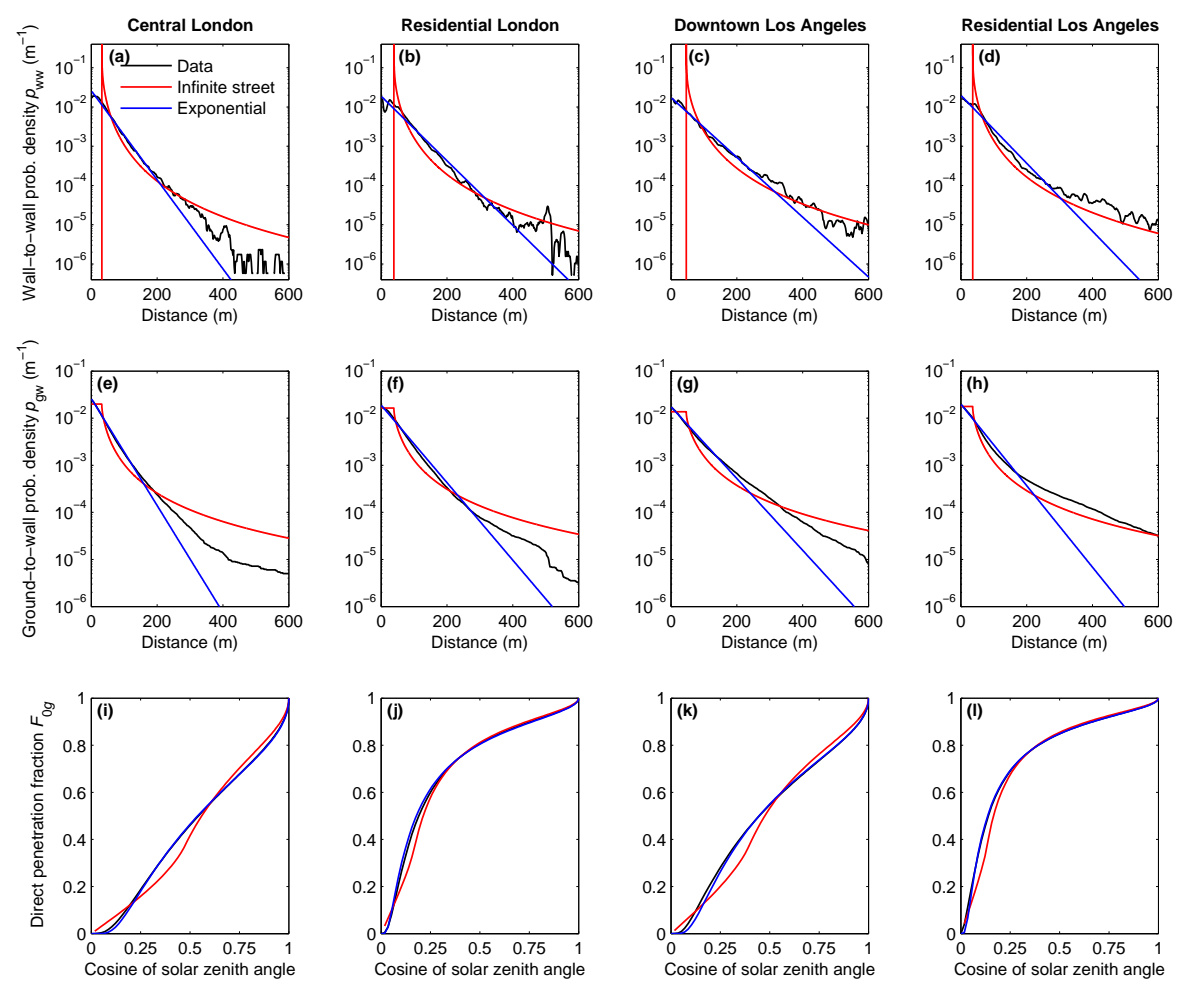

Fig. 6 (a-d) In black, the wall-to-wall probability distributions, $p_{w w}$, derived from the locations of the 'street, plaza, parking area or garden' category for the four scenes shown in Fig. 4. In red and blue, the fitted infinite-street and exponential models. (e-h) The corresponding ground-to-wall probability distributions, $p_{g w}$. (i-1) The corresponding direct penetration fraction $F_{0 g}$ as a function of the cosine of solar zenith angle.

for the two models, $W$ and $X$. We have chosen to select these lengths such that the diffuse ground-to-sky exchange factor, $F_{g s}$, is predicted exactly. This is achieved by numerically inverting (17) and (21) to obtain the values of $W$ and $X$ from the observed values of $F_{g s}$ and $H$; the values obtained by this method are shown in Table 1 . The associated analytical probability distributions for the two models (Eqs. 14, 15 and 19) are shown by the red and blue lines in Figs. 6a-6h. For all scenes, and for both $p_{w w}$ and $p_{g w}$, the exponential distribution fits much better than the infinite-street model for building separations between 0 and at least $200 \mathrm{~m}$. The infinite street is a particularly poor fit for $p_{w w}(x)$, predicting $p_{w w}=0$ for $x<W$, a delta function at $x=W$, and an underestimation by around a factor of two at $x \approx 200 \mathrm{~m}$. For larger building separations there is more variability between scenes, but arguably the infinite-street model fits a little better.

The red and blue lines in Figs. 6i-61 depict the predicted direct sky-to-ground exchange factor, $F_{0 g}$, revealing that the exponential model provides a better match to the values calculated from the real building distributions for all solar zenith angles. 


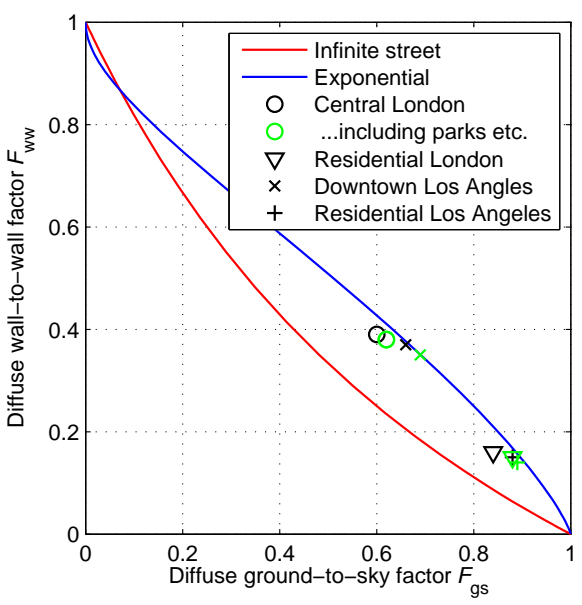

Fig. 7 Relationship between the diffuse wall-to-wall exchange factor $F_{w w}$ and ground-to-sky exchange factor $F_{g s}$ for the two analytic models (red and blue lines) and the four scenes depicted in Fig. 4 (black symbols). The green symbols depict the results from an alternative analysis of the four scenes in which parks, railways and major highways are added to the 'street' category.

This is because the probability distribution of building separations in the 0-200 $\mathrm{m}$ range, where the exponential model performs best, is more important for radiative exchange than larger building separations; indeed, only 1.0-3.9\% of $p_{w w}$ and 1.6$6.6 \%$ of $p_{g w}$ is contained in building separations greater than $200 \mathrm{~m}$.

In the case of diffuse exchange factors, the two models have already been fitted to ensure that $F_{g s}$ is predicted exactly, but $F_{w w}$ provides an independent point of evaluation. The lowest two rows of Table 1 show that the infinite-street model underpredicts $F_{w w}$ by on average $46 \%$, whereas the exponential model tends to overpredict $F_{w w}$ but by only $15 \%$ on average. This is analyzed in more detail in Fig. 7, which depicts the unique relationships between $F_{g s}$ and $F_{w w}$ predicted by the two analytical models. The black symbols show the corresponding values for the four real scenes. The poorer performance of the infinite-street model is due to $F_{w w}$ being particularly sensitive to $p_{w w}(x)$ for small $x$, where the two models are most different. Figure $3 \mathrm{c}$ also shows much lower $F_{w w}$ for the infinite-street than the exponential model for wall/ground area ratios in the range found in these four scenes $\left(0.26<A_{w} / A_{g}<1.4\right)$.

We now examine the impact of an alternative analysis of the four scenes, in which parks, railways and major highways are included in the 'street' category when deriving wall-to-wall and ground-to-wall probability distributions. The results are shown in Fig. 8, revealing that the probability distributions show somewhat higher tails for the larger building separations, but the fitted exponential model still fits better for separations of less than $200 \mathrm{~m}$, and also for the direct exchange factor shown in Figs. 8i-81. The green symbols in Fig. 7 show the $F_{g s}$ and $F_{w w}$ values for this alternative analysis, and again it is clear that the exponential model fits better.

If an urban radiation scheme using the exponential model were to be deployed in a weather or climate model then naturally the e-folding length $X$ would first need 

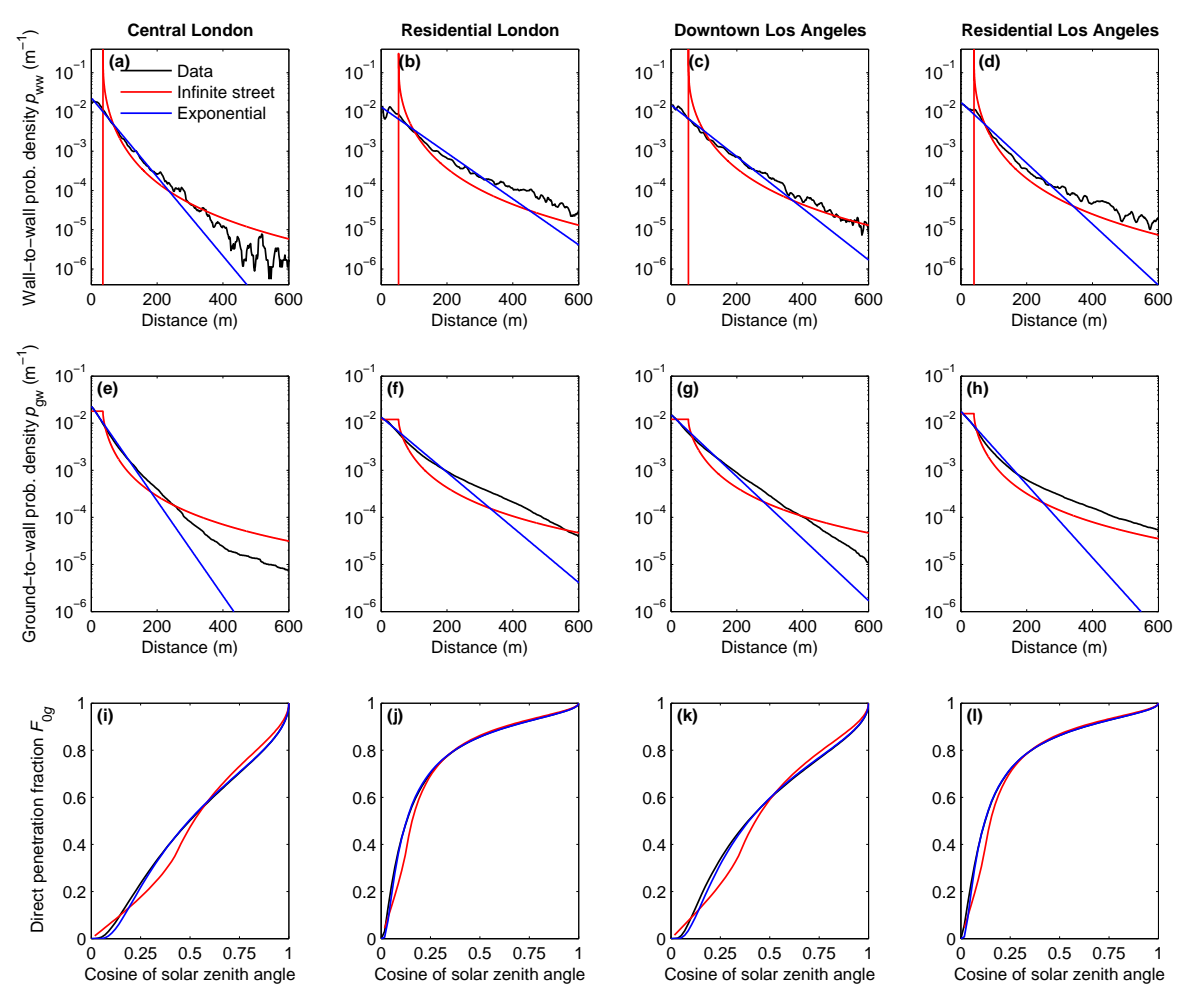

Fig. 8 As Fig. 6, but with parks, railways and major highways added to streets before performing the analysis.

to be estimated from the building layouts of a much larger number of cities. The strip method illustrated in Fig. 5 could of course be used to derive $p_{w w}$ and $p_{g w}$, but the inversion of the rather complex relation (21) to find the value of $X$ that predicts $F_{g s}$ (and hence $F_{g w}=1-F_{g s}$ ) exactly could be regarded as cumbersome. A simpler approach is to instead find the value of $X$ that predicts an approximate form of $F_{g w}$ in which $f_{g w}$ in (4) is replaced by an exponential of the form $f_{g w} \approx \exp (-x / Z)$, where $Z$ is a length scale to be defined. This leads to the following formula for estimating $X$ from an observed ground-to-wall probability distribution $p_{g w}$ :

$$
X \approx Z\left[\left(\int_{0}^{\infty} p_{g w} \mathrm{e}^{-x / Z} \mathrm{~d} x\right)^{-1}-1\right]
$$

When used with a length scale of $Z=1.5 H$, the estimated values of $X$ agree with those in Table 1 to within 1\%. Mean building height $H$ can be a somewhat ill-defined quantity in real cities, but we have found that using a fixed length scale of $Z=10 \mathrm{~m}$ also leads to acceptable results, with $X$ estimates then agreeing with those in Table 1 to within $1.2 \%$. 


\section{Discussion and conclusions}

In this paper it has been demonstrated that treating urban areas as streets of infinite length and constant width, as done in many weather and climate models, leads to significant errors in modelling the mean rates of exchange of solar and thermal-infrared radiation between the sky, walls and ground. Analysis of the probability distributions of wall-to-wall separation distances from real cities reveals that an exponential distribution is a good fit, and leads to a significantly better prediction of radiative exchange factors. Naturally, if this 'exponential model' of urban radiation were combined with an existing treatment of turbulent fluxes to create a full urban exchange scheme, care would need to be taken to ensure a consistent assumption about the areas of walls and ground. The exponential model for urban geometry could also be useful for other applications sensitive to building layout, such as blockage of mobile telephone signals (Bai et al., 2014).

While the radiative exchange formulas presented are a straightforward replacement for those in 'simple' existing urban radiation schemes (such as that described by Harman et al., 2004), an important question is how to incorporate the exponential model into more sophisticated schemes (e.g. Schubert et al., 2012; Krayenhoff et al., 2014; Redon et al., 2017) that represent vegetation and buildings of different height, yet are still underpinned by the infinite-street assumption. One approach could be to explore a useful property of the exponential model, which is that streams of radiation with a particular zenith angle in an urban canopy are attenuated according to the Beer-Lambert law, in the same way as light propagating through a turbid atmosphere. Equation 20 demonstrates this for direct solar radiation, but it is applicable to the entire radiation field if diffuse radiation is represented by a set of discrete zenith angles (e.g. Stamnes et al., 1988), an approach that underpins almost all one-dimensional multi-layer atmospheric radiative transfer schemes. This suggests that the infrastructure of such schemes could be adapted to the urban problem, enabling the prediction of the vertical profile of radiation within an urban canopy containing buildings of different heights, as well as the treatment of atmospheric absorption, emission and scattering. Note that it is ubiquitous for current urban radiation schemes to treat the space between buildings as a vacuum, but this is a dubious assumption in the thermal infrared.

In terms of vegetation, Hogan et al. (2018) used ideas from one-dimensional atmospheric radiation schemes to develop an accurate multi-layer model for treating radiation in forest canopies, embedded within which is the assumption that the horizontal separation of obstacles (which could be trees or buildings) follows an exponential distribution. This would therefore be an appropriate starting point for a more comprehensive urban radiation scheme that could accommodate street trees, atmospheric effects and multiple building heights. Naturally a crucial step is to evaluate any new urban radiation scheme using calculations on real urban geometry by explicit three-dimensional radiation models (e.g. Krayenhoff and Voogt, 2007; Gastellu-Etchegorry, 2008; Lindberg et al., 2008). 
Ordnance Survey Open Map with building height from lidar data collected in 2014 and 2015. Building geometry data for Los Angeles were obtained from the Los Angeles County GIS Data Portal, with the original data generated from aerial imagery. A number of the integrals were calculated using the online symbolic integration tools at www. wolframalpha.com and www. integral-calculator.com.

\section{References}

Bai T, Vaze R, Heath RW (2014) Analysis of blockage effects on urban cellular networks. IEEE Trans Wireless Comm 13:5070-5083

Gastellu-Etchegorry JP (2008) 3D modeling of satellite spectral images, radiation budget and energy budget of urban landscapes. Meteorol Atmos Phys 102:187-207

Grimmond CS, Oke TR (1999) Aerodynamic properties of urban areas derived from analysis of surface form. J Appl Meteorol 38:1262-1292

Grimmond CS, Blackett M, Best MJ, Barlow J, Baik J, Belcher SE, Bohnenstengel SI, Calmet I, Chen F, Dandou A, Fortuniak K, Gouvea ML, Hamdi R, Hendry M, Kawai T, Kawamoto Y, Kondo H, Krayenhoff ES, Lee S, Loridan T, Martilli A, Masson V, Miao S, Oleson K, Pigeon G, Porson A, Ryu Y, Salamanca F, Shashua-Bar L, Steeneveld G, Tombrou M, Voogt J, Young D, Zhang N (2010) The international urban energy balance models comparison project: first results from phase 1. J Appl Meteorol Climatol 49:1268-1292

Harman IN, Best MJ, Belcher SE (2004) Radiative exchange in an urban street canyon. Boundary-Layer Meteorol 110:301-316

Hogan RJ, Quaife T, Braghiere R (2018) Fast matrix treatment of 3-D radiative transfer in vegetation canopies: SPARTACUS-Vegetation 1.1. Geosci Model Dev 11:339-350

Johnson GT, Watson ID (1984) The determination of view-factors in urban canyons. J Clim Appl Meteorol 23:329-335.

Krayenhoff ES, Voogt JA (2007) A microscale three-dimensional urban energy balance model for studying surface temperatures. Boundary-Layer Meteorol 123:433-461

Krayenhoff ES, Christen A, Martilli A, Oke TR (2014) A multi-layer radiation model for urban neighbourhoods with trees. Boundary-Layer Meteorol 151:139-178

Kondo H, Genchi Y, Kikegawa Y, Ohashi Y, Yoshikado H, Komiyama H (2005) Development of a multilayer urban canopy model for the analysis of energy consumption in a big city: structure of the urban canopy model and its basic performance. Boundary-Layer Meteorol 116:395-421

Li D, Malyshev S, Shevliakova E (2016) Exploring historical and future urban climate in the Earth System Modeling framework: 1. Model development and evaluation. J Adv Model Earth Syst 8:917935

Lindberg F, Holmer B, Thorsson S (2008) SOLWEIG 1.0 - Modelling spatial variations of 3D radiant fluxes and mean radiant temperature in complex urban settings. Int J Biometeorol 52:697713

Martilli A, Clappier A, Rotach MW (2002) An urban surface exchange parameterisation for mesoscale models. Boundary-Layer Meteorol 104:261-304

Masson V (2000) A physically-based scheme for the urban energy budget in atmospheric models. Boundary-Layer Meteorol 94:357-397

Noilhan J (1981) A model for the net total radiation flux at the surfaces of a building. Build Environ $16: 259-266$

Redon EC, Lemonsu A, Masson V, Morille B, Musy M (2017) Implementation of street trees within the solar radiative exchange parameterization of TEB in SURFEX v8.0. Geosci Model Dev 10:385-411

Schubert S, Grossman-Clarke S, Martilli, A (2012) A double-canyon radiation scheme for multi-layer urban canopy models. Boundary-Layer Meteorol 145:439-468.

Sparrow EM, Cess RD (1970) Radiation Heat Transfer. Thermal Science Series, Brooks/Cole, Belmont CA

Stamnes K, Tsay SC, Wiscombe W, Jayaweera K (1988) Numerically stable algorithm for discreteordinate-method radiative transfer in multiple scattering and emitting layered media. Appl Opt 27:2502-2509

United Nations (2015) World Urbanization Prospects: The 2014 Revision. U.N. Department of Economic and Social Affairs, Population Division (ST/ESA/SER.A/366) 